\title{
A comparison of triple recording methods of eye movement
}

\author{
KAZUO KOGA \\ Division of Aerospace Psychology, Research Institute of Environmental Medicine, Nagoya University, \\ Chikusa-ku, . Vigugta $\$ 6 t$ \\ RYOJI OSAKA \\ Departnent of Psychology, Aichi-Gakuin L niversity, Aichi-gum, Aichi 470-01
}

\begin{abstract}
The aim of this article is to evaluate the simultaneous application of eye movement recording methods. The three methods of recording are the electro-oculogram (EOG), the corneal reflection method by a still camera (EMC) and the EMC aided by a computer (EMCC). Comparison of the methods in experimental applications was undertaken. A newly developed device and various improvements were made in the simultaneous recording experiment. The data of the EMCC were found more suitable for the analysis of fixations. Pattern analysis is easy with the EMIC method. The way to make the best use of their abilities is a combined use of them.
\end{abstract}

Key words: eve movement, corneal reflection method, image tracker, computer, EOG, saccade, fixation.

Many methods of recording eye movement have been developed in recent years. These efforts were focused on higher recording accuracy, more convenient operation and freedom of head movement (Young, 1976). The methods have distinctive features in experimental situations. Some are harmless to the subjects but of low accuracy, while others have very high accuracy but require rigid head immobilization.

Lehtela (1981) reported on research concerning these methodological problems. He used both an electro-oculogram and corneal reflection camera (NAC eye mark recorder). Osaka, Miwa, Sugiura, and Koga (1981) tried to compare three modes of recording simultaneously: the electro-oculogram, the corneal reflection methods by still camera and the computeraided corneal reflection method. In this latter study, it is unfortunate that the corneal reflection camera method was insufficient as an instrumental device. Thus only two different recording methods were compared strictly speaking.

The aim of this article is to show the distinctive features of three kinds of data recording methods in a simultaneous recording situation, i.e., the electro-oculogram (EOG), the corneal reflection method by still camera (eye movement camera, EMC), and the computer-aided automatic corneal reflected image processing method (EMC aided by computer, EMCG) which were used in combination. In our laboratory these three methods are normally used in different experimental situations independently. The EMC: method is the most well-known method of the three. Although quantitative analyses are difficult particularly as to the time sequence, the operation is convenient and the analysis of eye movement trace patterns can easily be done using this method. On the other hand, the EMCC method, which has been newly developed in our laboratory has an automatic tracking and digitizing function of the corneal reflection image, great accuracy and high speed sampling time. As for quantitative analysis, EMCG is the most suitable method. The development of the EMCC method was started about four years ago (Koga, 1979, 1980, 
1981, 1982; Osaka, Kida, Miwa, Koga, \& Sugiura, 1979). Several psychological data obtained from research using this system were published (Koga, Osaka, \& Miwa, 1980; Koga \& Osaka, 1982; Osaka \& Koga, 1980; Osaka, Kida Miwa, \& Koga, 1982). As to the EOG recording method, a high gain and low noise DC-amplifier and a depolarized high quality electrode is necessary. The high quality sintered type $\mathrm{Ag}-\mathrm{AgCl}$ electrode made in our own laboratory, were used (Osaka, Miwa, \& Taniguchi, 1982).

In this study these three modal recording methods were applied to the same eye movements simultaneously. The comparison of these methods will be discussed.

\section{Methods}

\section{Instruments}

EOG recording method. In the $\mathrm{EOG}$ measurments, $\mathrm{Ag}$ - $\mathrm{AgCl}$ sintered type electrodes and $\mathrm{K}^{+}$electrode paste (TDK Cio. Ltd.) were used. An analogue data recorder (TEAC, SR30) was used to record the data. Two DC-amplifier (NIHONKHODEN, NYSTAMOGRAPH) were used for amplifying the horizontal and vertical eye movements. The monitoring and temporal recording devices were heat pen oscilograph (SAN-EI, R8K) and an XY recorder (NATIONAL, VP-642A). Figure 1 illustrates the total paradigm of the experimental situation.

$E M C$ recording method. The special optical system was developed for the purpose of recording the corneal reflection image on film. Figure 2 shows an outline of the optical pathway and optical constructions. When the corneal reflection image from the corneal lamp was caught by the optical device, the optical pathway was divided into two by the beam splitter unit, one for the EMC recording instruments and the other for the EMCC recording system. This optical combination was constructed of five main parts: an objective optical lens unit which needs high magnification ratio, low distortional effect, easy focusing operation and large working distance (NIKKOR, Micro $55 \mathrm{~mm}$ and $2 \times$ rear compensator); an extention bellows for obtaining the highly magnified reflection image; a beam splitter unit; $35 \mathrm{~mm}$ still camera (NIKON, F3 with

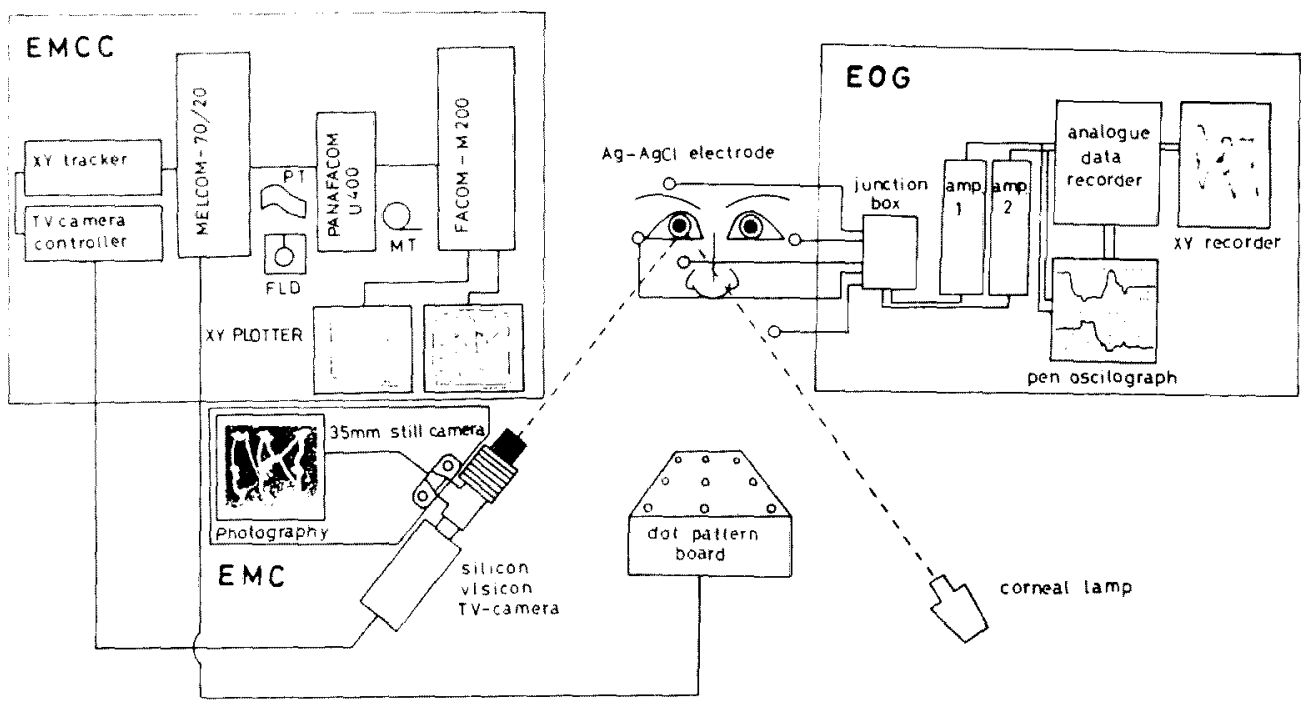

Fig. 1. Experimental paradigm of multiple recording measurements. The solid line means the connection between each device. 
motor driving unit); and a silicon visicon TV Camera for the mini-computer. The EMC recording system can also be seen in Fig. 1.

EMCC recording method. A part of the

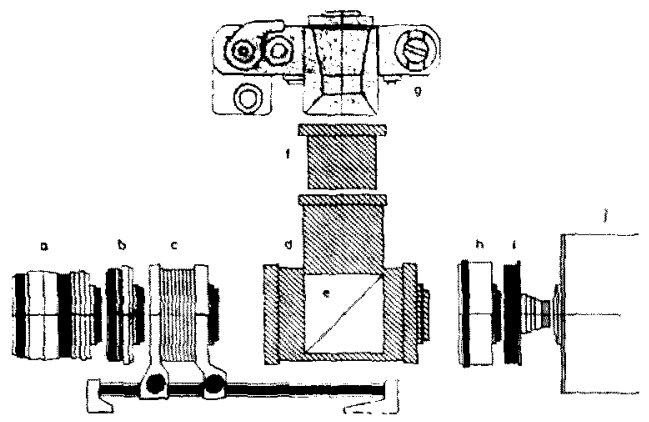

Fig. 2. Optical devices for the EMC and ENCC recordings. $(a, b \rightarrow$ objective lens combination, $\mathrm{c} \rightarrow$ extension bellows, $\mathrm{d}, \mathrm{e} \rightarrow$ beam splitter unit, $f \rightarrow$ focusing unit, $g \rightarrow 35 \mathrm{~mm}$ still camera, $h$, $\mathrm{i} \rightarrow$ mount conversion coupler, $\mathrm{j} \rightarrow$ silicon visicon TV camera.)
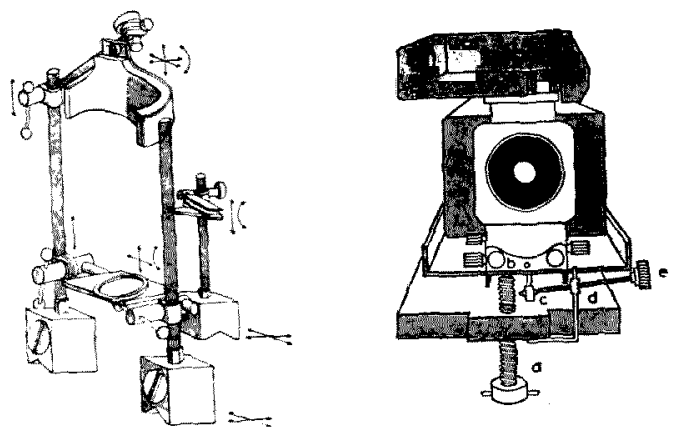

Fig. 3. The right figure shows the micro adjustment unit for optical system. (a $\rightarrow$ vertical adjustment, e $\rightarrow$ horizontal adjustment, b, c, $\mathrm{d} \rightarrow$ universal ball joint.) The left shows the universal headchin rest and bite board. Arrows in the figure indicates the adjustable direction of each parts. optical pathway of the corneal reflected image is caught by the silicon visicon TV camera which has the high sensitivity for the near-infrared (HAMAMATSU, C1000 and C1000-05). The TV signal was digitized to a two-dimensional scale by an XYtracker unit (HAMAMATSU, CI055). This digitizer has the ability for automatically detecting function. One of the brightest (or darkest) portions of the visicon sensor was converted to the display area for the digitized data with an absolute accuracy of 8-bit $\times 8$-bit in each $16.7 \mathrm{~ms}$ sampling time. The data of the corneal reflected image position from the XYtracker is stored in the CPU of a minicomputer (MITSUBISHI, MELCOM70/20). After finishing the experimental trial, the data was dumped on the flexible disk. As the maximum measuring time is 10 seconds, the 600 horizontal/vertical data (1/60 second sampling time over 10 seconds) were the maximum amount of recording capacity in the CPU. Nine kinds of application softwares for the aquisition and analysis of the data were developed. Two of them are most important. The application software called PROl was used for the aquisition of data and visual stimuli control. The other called D3 was used to convert the data for another large multi-purpose computer (FUJITSU, FACOM-M200) at the Nagoya University Computation Center.

General equipment for the experiment. The micro adjuster unit for the horizontal/ vertical adjustment of the optical unit is shown in Fig. 3. A universal adjustable head-chin rest and bite-board unit are also shown in Fig. 3. They are necessary for
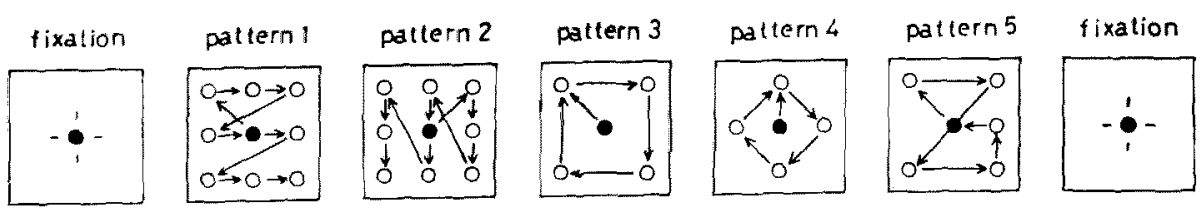

Fig. 4. Stimulus dot pattern which was presented to the subjects. The order of trial was conducted from left to right. 


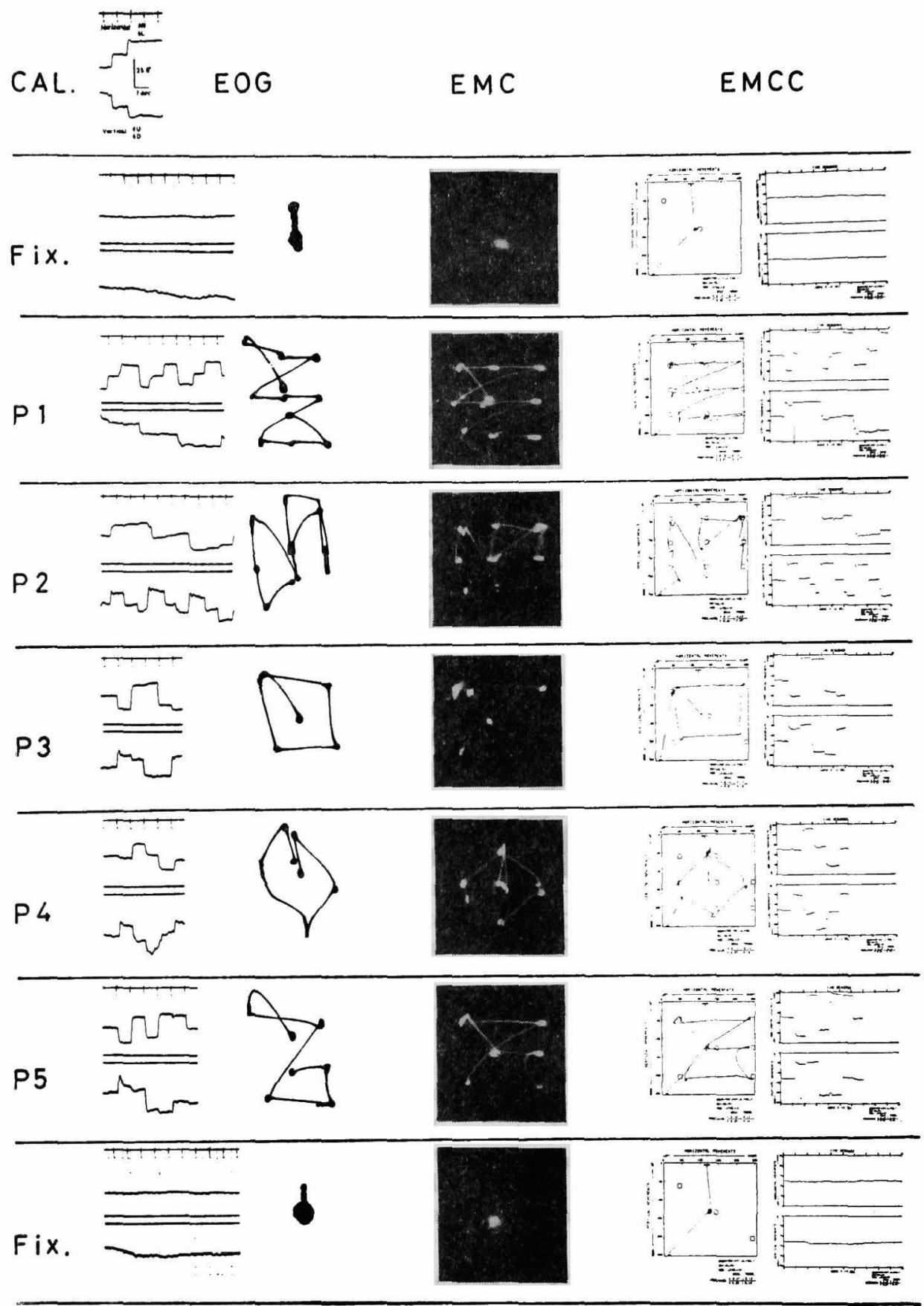

Subject's code; $82806 \mathrm{M}$

Fig. 5a. The comparison between three simultaneous recording methods to the seven stimulus patterns of subject $82804 \mathrm{M}$. Each column shows the different modal methods to the same eye movements and the row the seven kinds of stimulus patterns. 


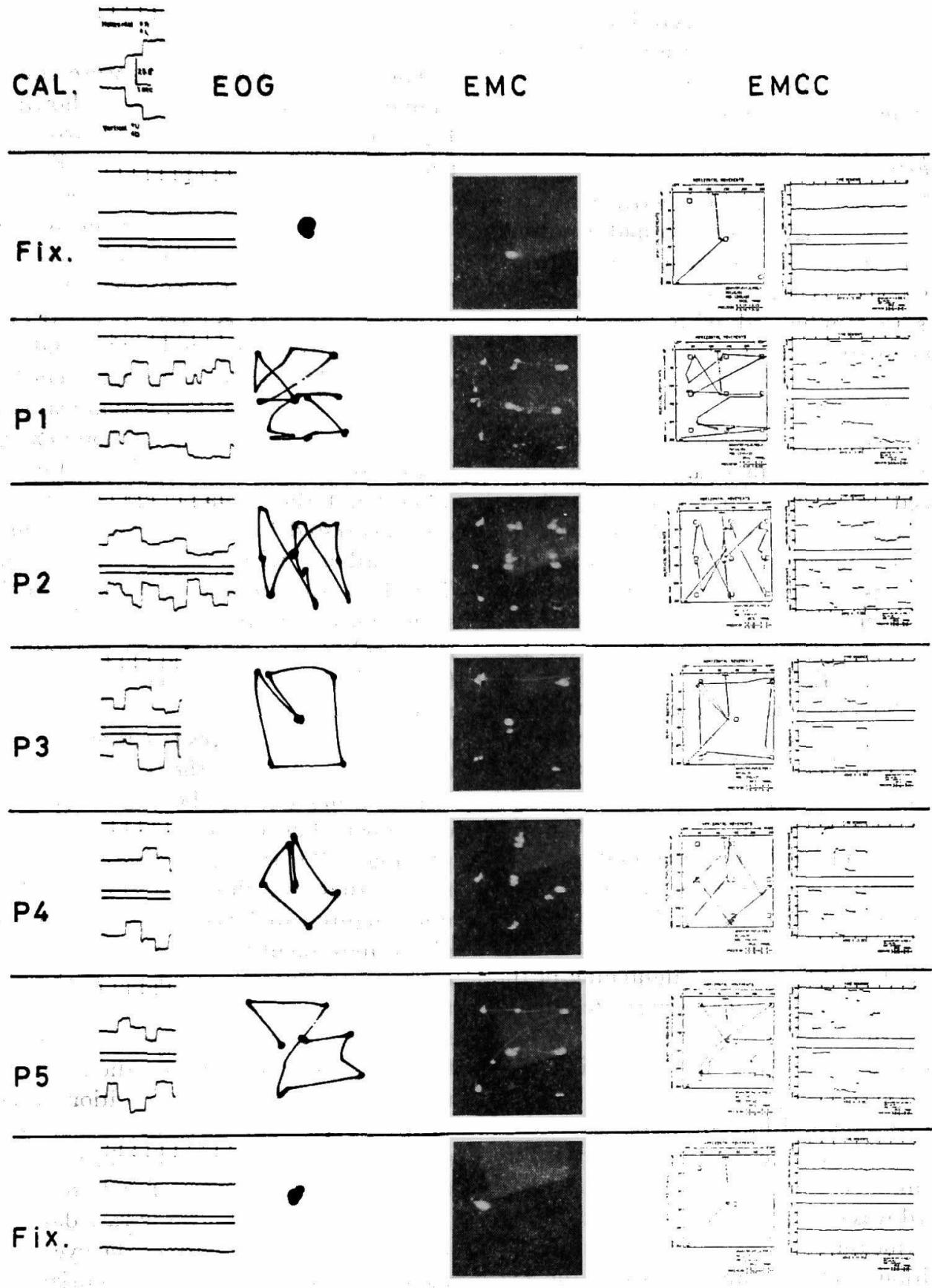

Subject's code; $82804 \mathrm{M}$

Fig. 5b. The same data of subject $82806 \mathrm{M}$. 
the highly accurate recording of the EMCC. The total apparatus was set on a stabilized desk to eliminating physical outer noise or vibration.

\section{Subjocts}

Two male students of Nagoya University $(82804 \mathrm{M}, 82806 \mathrm{M})$ who had a normal visual acuity were employed in the experiments. They were trained for four days to familialized with the apparatus and experimental situations.

\section{Procedures}

After the electrodes for the EOG measurement were attached, the subject was placed in a chair in a relaxed posture. The subject was instructed to place his head on the head-chin rest and to bite the bite-board. When the DC balance adjustment of the amplifier for the EOG, and optical focusing adjustment for the EMC and EMCC were completed, the calibration for determining the relationship between visual angles and eye positions was done. Subjects were instructed to gaze at the upper-left and bottom-right LED for three seconds each. During the calibration period, the horizontal / vertical coordinates corresponding to the visual angle was calculated. The calibration was done before each trial independently. After the calibration the lightening of the LED and measurements were started simultaneously. The stimulus dot pattern board with a $7 \times 7$ LED matrix in a $19.5 \mathrm{~cm}$ square plane was used. The exposure time and inter-stimulus-interval of sequential LED position was controlled by the mini-computer also. The dot pattern board was set in front of the subject's right eye (measured eye) of the primary eye position. The distance between the eye and the board was $42.5 \mathrm{~cm}$ with the matrix subtending a $25.8^{\circ}$ visual angle. Five sequential stimulus patterns which the subjects were shown are illustrated in Fig. 4. Each LED exposure was 1 second with no interval between exposures (pattern trial).

\section{Results}

The data of three trials were almost similar. The data which are shown in Figs. $5 \mathrm{a}$ and $5 \mathrm{~b}$ were an example from one trial. In the left two columns of Figs. 5a and $5 b$, the EOG data is shown both as a horizontal / vertical component pattern produced by a pen oscillograph and their compounded two dimensional patterns produced by the XY-recorder. In the latter case, to clarify the fixation and saccades, a little absorbent blotting recording paper were used. If the fixation was continued for a while, the fixated point can be identified as the blotted points. On the other hand the saccadic eye movements were expressed by the fine recorded lines in accordance with their saccadic speeds. The right two columns of the data in the same figure were plotted by the XY-plotting machine using the application software for the EMCG data. The software had two main functions. One was to produce the four kinds of figures based on the digitized data. Two of them are shown in Figs. $5 \mathrm{a}$ and $5 \mathrm{~b}$, the horizontal/vertical component figure and their compounded plottings. There are two other kinds of figures that are shown in Fig. 6. The upper figure which is called polar diagram of eye movement vectors indicates the direction and size of saccades. If the fixation are stable the size vector seems to be small. The bottom one which is called fixation contour-map means the cummulative histogram of the eye positions which stayed at the same positions. The more stable is fixation, the higher histogram is to be seen. The other function of the software is to normalize the raw data and calculate the total length of the eye movement pathway in each of the horizontal / vertical data and its compounded data. EMG data are shown only as two-dimensional eye movement trace patterns in the center column of Figs. $5 \mathrm{a}$ and $5 \mathrm{~b}$.

There are some differences in each modal measurement. The EOG measure- 
ments look like very smooth saccades. Micro eye movements or small shifts of eye are not represented. Sometimes a negative or a positive one directional potential shift was seen. It is uncertain whether this shift depends on the electrode's polarizational effect or the EOG potential shift itself. The amplitude of gain was controlled constantly in each horizontal and vertical channel at the time of data acqui-
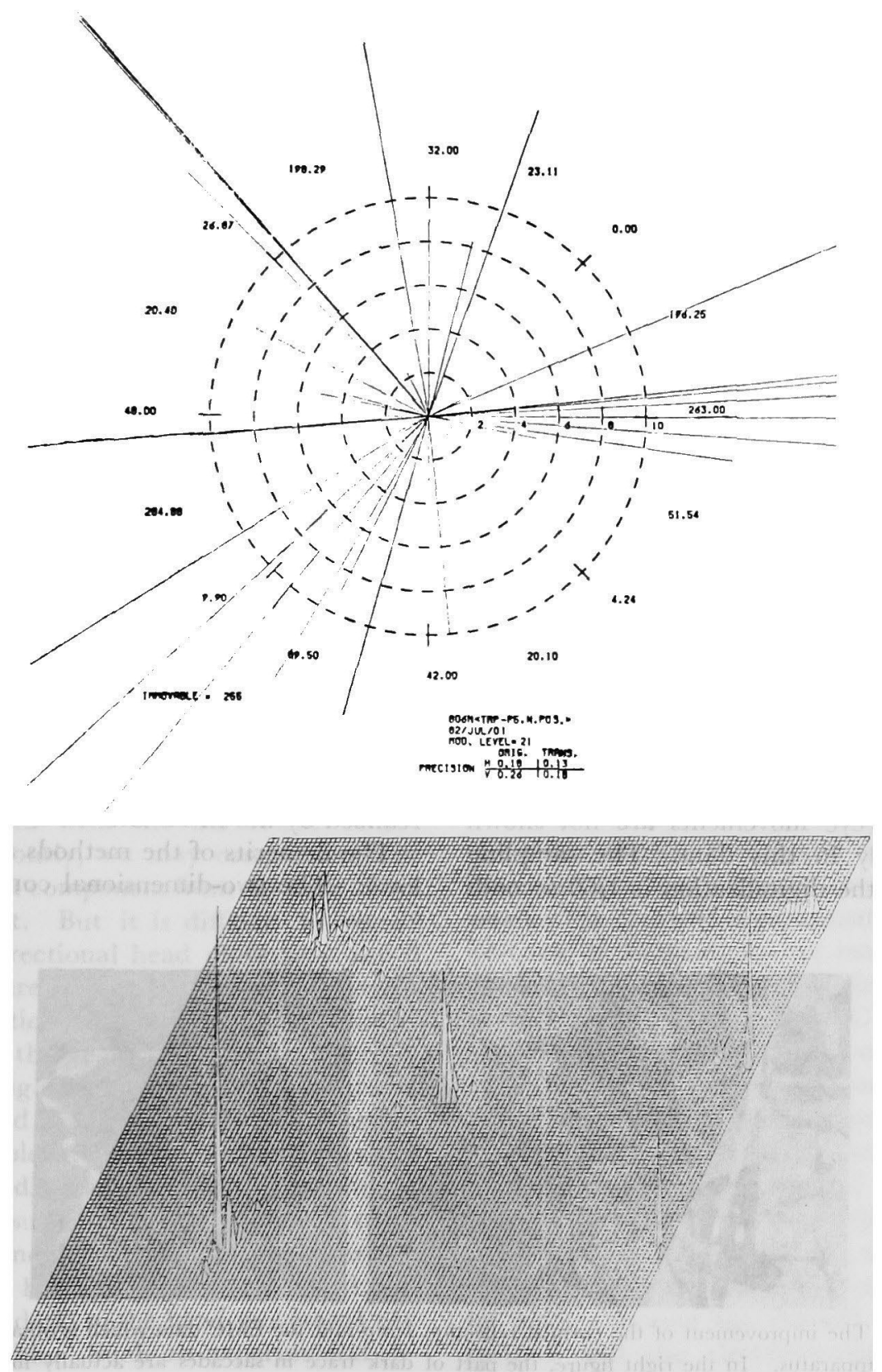

Fig. 6. The polar diagram which indicates the direction and the saccadic size in each $16.7 \mathrm{~ms}$, and the fixation contour-map which means the cummulative histogram of eye position. 
sition, and the output level of the data recorder was adjusted in accordance with the horizontal / vertical ratio of the calibration of the visual angle to compensate for the position difference of the electrodes. But there is a distortion of the visual trace pattern. It may depend on the effect of electrode position-mismatch which cannot be cancelled out by the adjustment of the output level. A study of the EOG vector potential map around the eye-ball will be necessary to measure the multiple position potentiality of the EOG simultaneously. If the map is standarized, the position at which the electrodes are to be attached can be determined instantly. Another way of solving this problem is to go through the digital transformation of the $\mathrm{A} / \mathrm{D}$ converted EOG data according to the accurate horizontal and vertical eye movements. If the $\Lambda / D$ conversion of the EOG is done in the accurate measurement condition, the distortion of the eye movement trace pattern which is due to the electrode's position mismatch can be corrected by the transformational procedure of data.

The EMCC measurement data shows both the horizontal/vertical component individually and the compounded figure. Saccadic eye movements are not shown completely in this data. The sampling time for the digitalization is $1 / 60$ seconds which is not so sufficiently fast enough to record the large saccades. A faster sampling time over about $1 / 300$ seconds or more will be needed. But the EMCG measurement system is powerful enough to measure micro eye movements during fixation. In Figs. $5 a$ and $5 b$, the data of the fixation trials of the EOG and EMC are not indicated in detail. On the other hand, the EMCC recordings of fixation show very small eye movements about $6^{\prime} / 16.7 \mathrm{~ms}$ of accuracy. Higher accuracy of about $2^{\prime}$ $16.7 \mathrm{~ms}$ or more using another optical combination is achieved now in our laboratory.

\section{Discussion}

Merits and demerits exist in each method. The merits are as follows. Quantitative analysis is possible with the EMCC method because of the digital data form. The main purpose of this article lies in the comparison of three different methods of eye movement recordings, so the digital data analysis was not done. The description of small or micro eye movement during fixation are more clearly described by the EMCG method than by the EMC or EOG methods. The finest recordings of high speed eye movement of saccades are realized by the EMC and EOG methods.

The demerits of the methods are as follows: The two-dimensional compounded
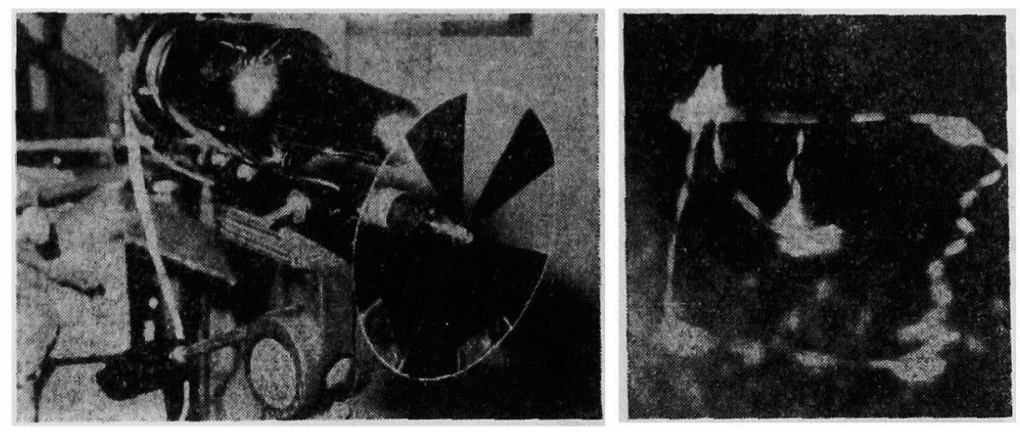

Fig. 7. The improvement of the corneal reflection lamp and the EMC data which is obtained by the improved apparatus. In the right figure, the part of dark trace in saccades are actually in red colour, and bright ones in saccades and fixations are in tungsten colour. A dark or bright part of the stripe shows the $4.5 \mathrm{~ms}$ saccadic time. The subject's code is $82807 \mathrm{M}$. 
description of horizontal / vertical is insufficient in the EOG method because of the DC potential shift that is affected perhaps by the polarization of the electrodes. The drift of the baseline of potential became an obstacle in distinguishing between the real eye movement and another exterior artifacts. The EMC data is represented in the form of compounded figure on the film. If the fixation is unstable, the area of recorded data will be larger in comparison with that of the stable fixation. The total length of eye movement trace cannot be measured by this method. On the other hand, for the recording of saccadic eye movements, the EMC method is more suitable than the EOG or EMCC. method in the pattern form. The recording of high speed saccades over $30^{\circ}$ or more will be realized by the EMC method. The quantitative analysis during fixation is a weak point for the EOG and the EMC methods. As to the saccades, sometimes its speed slows down, as in the case of tiredness or when approaching the terminal point of fixation. In such a case, the EMCC catch the saccades accidentally.

Generally speaking, the EOG method can measure eye movement without head immobilization. If the head movement is recorded with the movement of eye simultaneously, we may cancel the head movement component from the data of eye movement. But it is difficult to record the all-directional head movement and if the measurement can be achieved, there is no theoretical transformational formula to calculate the real eye movement vectors eliminating the head movement vectors. If the head immobilization is not possible, for example in the case of infants or the clinically disordered, the EOG method is the most suitable strategy. In our study head immobilization was done strictly using the head-chin rest, bite-board and stabilized desk, so the head movement can be negligible. Such an experimental situation may be suitable for the normal adult subjects only.
As to the quantitative analysis of the EMC, it has been suggested that a time sequential analysis by improving the corneal reflection lamp is possible. To realize this potential, some improvements has been made in the experimental apparatus, as shown in Fig 7. A wheel divided into ten sectors, red and transparent, which are set in front of the corneal reflection lamp, can be rotated at 1333 $\mathrm{rpm}\left(\mathrm{min}^{-1}\right)$. The beam of the corneal lamp are changed from red to noncoloured in $4.5 \mathrm{~ms}$ alternatively. Consequently, the saccadic trace on the film is made in red and non-red stripes sequentially. In this case, the film needs a high sensitivity above ISO 1600 or more to get the normal exposure. The sample data obtained from this method is shown in Fig. 7. A parts of dark trace in saccades were in red, actually, and the bright ones in saccades and fixations are tungsten coloured. The faster the saccade come to, the lower the frequency of stripes in the saccades. The saccades of the horizontal direction seems to be smoother than that of vertical. The saccadic speed of horizontal movements seems faster than that of vertical. Time sequential analysis can be done by this method in the EMC method.

The conclusion from the comparison of the three method is reduced as follows. The multiple measurement can compensate for the demerits of each method. The best way to measure the eye movement is to apply the combined use of them. The best combination is the EMC and the EMCC methods while fixation can be recorded by the EMCC method more exactly. The EOG method can compensate for both the demerits of the EMC and EMCC methods. But the accuracy of the EOG is not so great. To test the reliability of the corneal reflection method, simultaneous application of the EOG method must be made, because the corneal reflection method and the electro-oculogram can measure the different aspects of the same eye movement. 


\section{References}

Koga, K. 1979 Computcrized tracking system for the studies of eye movement camera and open field experiments. Annuals of the $R e$ search Institute of Environmental Medicine, Nagoya University, 30, 83-89. (In Japanese)

Koga, K. 1980 Data processing system of the eye movemens by MELCOM-70/20 and FACOMM200 systems. Annuals of the Research Institute of Environmental Medicine, Nagoya University, 31, 8793. (In Japanese)

Koga, K. 1981 Data processing system of the eyc movement by MELCOM-70/20 and FACOMM200 system (II). Annuals of the Research Institute of Environmental Medicine, Nagoya University, 32, 71-74. (In Japanese)

Koga, K. 1982 Data processing system of the eye movement by MELCOM-70/20 and FACOMM200 system (III). Annuals of the Research Institute of Environmental Medicine, Nagoya University, 33, 87-90. (In Japanese)

Koga, K., \& Osaka R. 1982 Methodological study on the measurements of eye movement (IV) -The matrix map of eye movement during fixations in each cye position-. Annuals of the Research Institute of Environmental Medicine, Nagoya University, 33, 87-90. (In Japanese)

Koga, K., Osaka, R., \& Miwa, T. 1980 The study of fixations during thinking processes. Annuals of the Research Institute of Environmental Medicine, Nagoya Univeristy, 31, 68-71. (In Japanese)

Lehtela, J. 1981 Differences in eye movement data recorded by electro-oculography and cor- neal reflection techniques. Human Factors, 23, 661-665.

Osaka, R., \& Koga, K. 1980 Instability of ocular control during mental tasks. Environmental Medicine, 25, 1-13.

Osaka, R., Miwa, T., \& Taniguchi, M. 1982 Fabrication of $\mathrm{Ag}-\mathrm{AgCl}$ electrode and its thermal adaptation to skin temperature. Annuals of the Research Instilute of Environmental Medicine, Nagoya University, 33, 104-107. (In Japanese)

Osaka, R., Kida, M., Miwa, T., \& Koga, K. 1982 Analysis of visual fixation by eye-deviation and single saccades. Annuals of the Research Instilute of Environmental Medicine, Nagoya University, 33, 7781. (In Japanese)

Osaka, R., Miwa, T., Sugiura, K., \& Koga, K. 1981 Methodological study on the measurements of eye movement (II) --Simultaneous recording by corneal reflection method and electro-oculograph-. Annuals of the Research Institute of Environmental Medicine, Nagoya University, 32, 77-81. (In Japanese)

Osaka, R., Kida, M., Miwa, T., Koga, K., \& Sugiura, K. 1979 Eye movement and stability of visual space. Annuals of the Research Institute of Environmental Medicine, Nagoya University, 30, 60-65. (In Japanese)

Young, L. R. 1976 Physical characteristics of the eyc used in eye movement measurement. In R. A. Monty \& J. W. Senders (Eds.), Eye movements and psychological processes. New York: John Wilcy \& Sons. Pp. 157-202.

(Received April 11 1983; accepted Sept. 11, 1983) 